\title{
Prevalence and antibiotics susceptibility patterns of carbapenem resistant Enterobacteriaceae
}

\begin{abstract}
Enterobacteriaceae is a family of Gram negative rods. However it natural habitat in the intestinal tract of humans and animals, but it has been implicated in many human diseases. The emergence of antimicrobial resistance among Enterobacteriaceae isolates has been increasingly reported worldwide and has become a major threat to the provision of healthcare. Carbapenems is beta-lactam antibiotics which is considered as a last line of therapy for multidrug resistant. The occurrence of carbapenem resistance among Enterobacteriaceae is a major health challenge which reduce the antibiotics choices that use to treat the infections which cause by these bacteria. This review was focused to increase our understanding about carbapenem resistance; and to display the size and extent of this problem base on up to day published works. The prevalence of carbapenem resistance Enterobacteriaceae (CRE) is slightly different among different countries, and their resistance rate for commonly uses antibiotics has been significantly detected. The use of combined antibiotics seem to be is only up to day known therapeutic choice. The major worrisome, treatment of the infections causes by these multidrugs resistant organisms is extremely difficult which may results in high mortality rates and healthcare costs. We need to focus on continues searching for other highly effective and low cost alternatives therapies.
\end{abstract}

Volume 6 Issue 3 - 2018

\begin{abstract}
Babiker Saad Almugadam,' Nmariq Osman Ali, ${ }^{2}$ Alaaeldeen Balal Ahmed, ${ }^{3}$ Elnaim Bushra Ahmed, ${ }^{4}$ Lili Wang ${ }^{5}$

'Department of Microbiology, University of El Imam El Mahdi, Sudan

${ }^{2}$ Department of Microbiology, Elrazi University, Sudan

${ }^{3}$ Department of Microbiology, White Nile University, Sudan ${ }^{4}$ Department of Medical Laboratory Investigations, Kosti Police Hospital, Sudan

${ }^{5}$ Department of Microecology, Dalian Medical University, China
\end{abstract}

Correspondence: Babiker Saad Almugadam, Department of Microbiology, Faculty of Medical Laboratory Sciences, University of Elimam Elmahdi Kosti city, Sudan, Tel 002499 177I 7034, Email Babiker888@yahoo.com

Received: April 17, 2018 | Published: May 23, 2018

Keywords: antimicrobials resistant, carbapenemase producing Enterobacteriaceae, carbapenem resistant Enterobacteriaceae, clinical isolates, prevalence

\section{Introduction}

Enterobacteriaceae is a family of Gram negative rods, facultative anaerobes, ferment a wide range of carbohydrates, possess a complex antigenic structure, and produce a variety of toxins and other virulence factors. ${ }^{1,2}$ Natural habitat in the intestinal tract of humans and animals. ${ }^{2}$ It is more than 40 genera and 150 species. Only few genera were consider as true pathogens (Escherichia, Salmonella, Shigella, and Yerssina), and few are opportunistic pathogens (Klebsiella, Citrobacter, Enterobacter, Proteus, Provodencia, Morganella, Serritia, and Edwardsella). ${ }^{2,3}$ Different members of Enterobacteriaceae has been known as a causatives of different intraintestinal (as diarrhea) and extra-intestinal diseases includes wound infections, pneumonia, septicemia, bacteremia, and meningitis. ${ }^{2}$

The emergence of antimicrobial resistance among Enterobacteriaceae has been increasingly reported worldwide and has become a major threat to the provision of healthcare. ${ }^{1}$ The resistance of carbapenems are increased dramatically worldwide and recently among members of Enterobacteriaceae family. ${ }^{4}$

Carbapenems is beta-lactam antibiotics (includes imepenem, meropenem, ertapenem and doripenem) which is considered as a last line of therapy for multidrug resistant (MDR). ${ }^{5,6}$ Carbapenem resistance has been detected and considered as a one of major health problem worldwide and limit the choice of selected antibiotics therapies to treat bacterial infections. ${ }^{7}$ Carbapenem resistance may result either from production of carbapenemase that breakdown carbapenem or production of beta-lactamase (ESBLs or Ampc) together with porin loss. Carbapenemase producing enterobacteriaceae (CPE) produce carbapenemase which is a main mechanism of carbapenem resistance. ${ }^{8}$ Carbapenemase was classified molecularly to three classes (A, B, and D) ${ }^{4,8}$ K. pneumoniae carbapenemase (KPC) which belong to molecular class A, OXA48 (class D), and New Delhi metallo betalatamase (class B) are the most common carbapenemases produce by Enterobacteriaceae family. ${ }^{4,8}$ These enzymes (K. pneumoniae carbapenemase, OXA48, and New Delhi metallo beta-latamase) confer resistance to virtually all beta-lactam agents, including penicillins, cephalosporins, monobactams, and carbapenems. ${ }^{4}$ Detection of carbapenem resistance can be perform phenotypically or genotypically using molecular techniques. ${ }^{9,10}$ This review was focused to increase our understanding about carbapenem resistance; and to display the size and extent of this problem base on up to day published works.

\section{Prevalence of CRE}

Understanding the prevalence of CRE is necessary to provide information on the temporal, and geographic occurrence of carbapenem resistance; and the size of this problem in order to facilitate its prevention and control. Base on previously published researches, the prevalence's of CRE among clinical samples were slightly different among different regions in the world.

\section{Africa}

The emergence of CRE has been observed and reported in many studies includes Oduyebo OO et al. ${ }^{11}$ (Nigeria), Legese $\mathrm{MH}$ et al. ${ }^{12}$ 
(Ethiopia), Okoche D et al. ${ }^{13}$ (Uganda), Amer WH et al..$^{14}$ (Egypt), Camara A et al. ${ }^{15}$ (Senegal), and Wartiti MA et al. ${ }^{16}$ (Morocco) which reported the prevalence of CRE were $15.2 \%, 12.12 \%, 28.6 \%, 62.7 \%$, $5.1 \%$, and $2.8 \%$ respectively. ${ }^{11-16}$

\section{Asia}

In Asia, the prevalence of CRE among clinical samples was determined in many studies includes Rao A et al. ${ }^{17}$ (India), Amjad A et al. ${ }^{18}$ (Pakistan), Jamal WY et al. ${ }^{19}$ (Kuwait), Li Y et al. ${ }^{20}$ (China), Kandeel A et al. ${ }^{21}$ (Saudi Arabia), Nair PK et al..$^{22}$ (India), Zaidah A et al. ${ }^{23}$ (Malaysia), Jan $\mathrm{R}$ et al. ${ }^{24}$ (South India) and Khare $\mathrm{V}$ et al. ${ }^{25}$ (India) which reported the prevalence of CRE were $13.95 \%, 69 \%$, $8 \%, 18.1 \%, 1.77 \%, 12.26 \%, 5.76 \%, 8 \%$ and $37.9 \%$ respectively. ${ }^{17-25}$

\section{Europe, Australia, and America (North and South)}

The occurrence of CRE has been investigated in many studies includes Huang $\mathrm{T}$ et al. ${ }^{26}$ (Belgium), Baran I et al. ${ }^{27}$ (Turkey), Mathersa J et al..$^{28}$ (USA), Pfaller MA et al. ${ }^{29}$ (Latin America), Partina I et al. ${ }^{30}$ (Russia), Pfaller MA et al. ${ }^{31}$ (Australia and New Zealand) and Logan LK et al. ${ }^{32}$ (USA) which reported the frequency of CRE were $3.46 \%, 2.8 \%, 5.7 \%, 6.6 \%, 11.6 \%, 0.1 \%$ and $0.08 \%$ respectively. ${ }^{26-32}$ Also Sader HS et al. ${ }^{33}$ study were found the occurrence of CRE in Poland, Italy, Greece and Romania were $17.3 \%, 7.5 \%, 7.4 \%$, and $5.0 \%$ respectively. ${ }^{33}$

\section{Antibiotics susceptibility patterns of CRE}

All members of carbapenem resistance Enterobacteriaceae showed high resistant rate for all or most commonly uses penicillins, cephalosporins, monobactams, and quinolones as it reported by many studies. ${ }^{34-40}$ The antibiotics which has a good activity on most CRE isolates were fosfomycin, tigecycline, polymyxin, amikacin, gentamycin and colistin. ${ }^{33,41-47}$

Many studies suggests the use of combined therapies to treat CRE. Falagas ME et al. ${ }^{48}$ study suggests the use of combined antibiotics may offer a comparative advantage over monotherapy. ${ }^{48}$ Nabarro LB et al. ${ }^{49}$ Study conclude there is increasing evidence to support the use of combination therapy to treat infections that cause by CRE. ${ }^{49}$ Fredborg $\mathrm{M}$ et al. ${ }^{50}$ study conclude meropenem triple combinations with a polymyxin and rifampin exhibited highest synergistic activity against carbapenem producing Enterobacteriaceae. ${ }^{50}$

\section{Discussion}

Carbapenem is a one of antibiotics that offer broad spectrum activity and use as a last line therapy for multidrug resistant bacteria. The treatment of infections causes by drugs resistance bacteria is sometime impossible and may lead to unexpected or bad complications. Antimicrobial resistance increases the cost of health care, and possibility of complications. Without effective antimicrobials for prevention and treatment of CRE infections, medical procedures become very high risk. The major worrisome, treatment of the infections causes by these multidrug organisms is extremely difficult which may results in high mortality rates and healthcare costs.

In this review, we found carbapenem resistance has been emerged worldwide and is beginning to spread. This emergence might result from the absence of public health surveillance programs in most countries that hided this problem, so the public health surveillance programs must be establish in all countries to facilitate the discovery of problems as early as possible. Also new alternatives therapies need to be developed to encounter bacteria with this kind of resistance; and the hospitals need excellent infection treatment and control to prevent the morbidity and spread of CRE.

Also we found the prevalence of CRE in African countries are slightly low when compare with Asian countries; and in Australia and American countries is slightly low when compare with African countries. While the prevalence of CRE in European countries seem to be same in African. Also there is a marked reduction in the susceptibility of carbapenem resistance isolates to anther antibiotics. This differences in the prevalence of CRE and their susceptibility to antibiotics among the isolates of different world countries may arise from the different in geographical location and poor infection control in health care settings or the misuse and availability of non-prescribed therapies that describe by WHO as a accelerators of drugs resistant.

As we found the use of combined therapies up to day is only known appropriate and highly effective choice for treatment of CRE infections. But the use of combined therapies lead knock down the beneficial flora in the body and may associate with many serious diseases or unexpected complications. So the looking for anther alternatives therapies may be a one of the solutions for this problem.

\section{Conclusion}

The prevalence of CRE has been emerged worldwide. As we displayed there is a significant rate of carbapenem resistance among Enterobacteriaceae. Formulating an antimicrobial policy with its strict implementation and regular surveillance must be establish. Further studies need to focus on continues searching for a highly effective, low cost, and minimum side effect alternatives therapies.

\section{Acknowledgements}

None.

\section{Competing interests}

We declare that we have no competing interests.

\section{References}

1. Toolkit. Facility guidance for control of carbapenem-resistant enterobacteriaceae (CRE). CDE; 2015.

2. Koneman EW, Allens SO, Janda WM, et al. Colour atlas and textbook for diagnostic microbiology. Enterobactericeae. JB Lippincott Company, Philadelphia; 2006. p. 61-402.

3. Don JB, Noel RK, James TS. Bergey's manual of systematic bacteriology. In: George MG, editor. The Gammaproteobacteria. Williams \& Wilkins, New York: Springer; 2005. p. 1108.

4. Souli M, Galani I, Antoniadou A, et al. An outbreak of infection due to beta-lactamase Klebsiella pneumoniae carbapenemase-2 producing K. pneumoniae in a Greek University Hospital: molecular characterization,epidemiology, and outcomes. Clin Infect Dis. 2010;50(3):364-373.

5. Hu F, Chen S, Xu X, et al. Emergence of carbapenem resistant clinical Enterobacteriaceae isolates from a teaching hospital in Shanghai, China. J Med Microbiol. 2012;61(Pt 1):132-136.

6. Sidjabat H, Nimmo GR, Walsh TR, et al. Carbapenem resistance in Klebsiella pneumoniae due to the New Delhi Metallo B-lactamase. Clin Infect Dis. 2011;52(4):481-484.

7. Tzouvelekis L, Markogiannakis A, Psichogiou M, et al. Carbapenemases 
in Klebsiella pneumoniae and Other Enterobacteriaceae. CMR 2012;25(4):682-707.

8. Glasner C, Albiger B, Buist G, et al. European survey on carbapenemaseproducing Enterobacteriaceae (EuSCAPE) working Group: carbapenemase-producing Enterobacteriaceae in Europe: a survey among national experts from 39 countries, February 2013. Euro Surveill. 2013;18(28):20525.

9. Van Dijk K, Voets GM, Scharringa J, et al. Disc diffusion assay for detection of class A, B and OXA-48 carbapenemases in Enterobacteriaceae using phenyl boronic acid, dipicolinic acid and temocillin. Clin Microbiol Infect. 2014;20(4):345-349.

10. Arend LN, Pilonetto M, Siebra CA, et al. Phenotypic and molecular characterization of 942 carbapenem-resistant Enterobacteriaceae (CRE) in southern Brazil. J Infect Chemother. 2015;21(4):316-318.

11. Oduyebo OO, Falayi O M, Oshun P, et al. Phenotypic determination of carbapenemase producing Enterobacteriaceae isolates from clinical specimens at a Tertiary Hospital in Lagos, Nigeria. Niger Postgrad Med J. 2015;22(4):223-227.

12. Legese MH, Weldearegay GM, Asrat D. Extended-spectrum betalactamase and carbapenmase-producing Enterobacteriaceae among Ethiopian children. Infect Drug Resist. 2017;10:27-34.

13. Okoche D, Asiimwe BB, Katabazi FA, et al. Prevalence and characterization of carbapenem-resistant Enterobacteriaceae isolated from Mulago National Referral Hospital, Uganda. PLoS One 2015;10(8):e0135745.

14. Amer WH, Khalil HS, Abd El Wahab MAA. Risk factors, phenotypic and genotypic characterization of carbapenem resistant Enterobacteriaceae in Tanta University Hospitals, Egypt. Int J Infect Control. 2016;12:1.

15. Camara A, Mane MT, Ba-Diallo A, et al. Extended-spectrum betalactamase and carbapenemase-producing Enterobacteriaceae clinical isolates in a Senegalese teaching hospital: A cross sectional study. Afr $J$ Microbiol Res. 2016;11(44):1600-1605.

16. Wartiti MA, Bahmani FZ, Elouennass M, et al. Prevalence of carbapenemase producing Enterobacteriaceae in a University Hospital in Rabat, Morocco: A19-months prospective study. The international arabic journal of antimicrobial agents. 2012;2(3):4.

17. Rao A, Indumathi VA. Detection of carbapenem resistant enterobacteriacae from clinical isolates. Int J Curr Microbiol App Sci. 2016;5(5): 864-869.

18. Amjad A, Mirza IA, Abbasi SA, et al. Modifid hodge test: A simple and effective test for detection of carbapenemase production. Iran $J$ Microbiol. 2011;3(4):189-193.

19. Jamal WY, Albert MJ, Rotimi VO. High prevalence of New Delhi Metallo- $\beta$ - Lactamase- 1 (NDM-1) producers among carbapenemresistant Enterobacteriaceae in Kuwait. PLoS One. 2016;11(3):e0152638.

20. Li Y, Shen Y, Zhang Y, et al. Rapid increase in prevelence of carbapenemresistant Enterobacteriaceae (CRE) and emergence of colistin resistance gene mor- 1 in CRE in a hospital in Henan, China. $J$ Clin Microbiol. 2018;56(4);1932-1937.

21. Kandeel A. Epidemiology of carbapenemase producing Enterobacteriaceae in a general hospital. J Microbiol Infect Dis. 2015;5(2):57-62

22. Nair PK, Vaz MS. Prevalence of carbapenem resistant Enterobacteriaceae from a tertiary care hospital in Mumbai, India. JMID. 2013;3(4):207-210

23. Zaidah A, Mohammad N, Suraiya S, et al. High burden of carbapenemresistant Enterobacteriaceae (CRE) fecal carriage at a teaching hospital: costeffectiveness of screening in low-resource setting. Antimicrobial Resistance \& Infection Control. 2017;6:42.
24. Jan R, George N, Mathew M, et al. Prevalence of carbapenem resistant enterobacteriaceae in a tertiary care referral centre: Kerala, South India. International Journal of Current Research. 2016;8(12):44353-44355.

25. Khare V, Gupta P, Haider F, et al. Study on MICs of tigecycline in clinical isolates of carbapenem resistant Enterobacteriaceae (CRE) at a tertiary care centre in North India. J Clin Diagn Res. 2017;11(3):DC18-DC21.

26. Te-Din H, Catherine B, Pierre B, et al. Prevalence and mechanisms of resistance to carbapenems in Enterobacteriaceae isolates from 24 hospitals in Belgium. J Antimicrob Chemother. 2013;68(8):1832-1837.

27. Baran I, Aksu N. Phenotypic and genotypic characteristics of carbapenemresistant Enterobacteriaceae in a tertiary-level reference hospital in Turkey. Ann Clin Microbiol Antimicrob. 2016;15:20.

28. Mathersa J, Coxa HL, Kitchelb B, et al. Molecular dissection of an outbreak of carbapenem-resistant enterobacteriaceae reveals intergenus KPC carbapenemase transmission through a promiscuous plasmid. mBio. 2011;2(6):e00204-e00211.

29. Pfaller MA, Shortridge D, Sader HS, et al. Ceftolozane-tazobactam activity against drug-resistant Enterobacteriaceae and Pseudomonas aeruginosa causing healthcare-associated infections in Latin America: report from an antimicrobial surveillance program (2013-2015). Braz J Infect Dis. 2017;21(6):627-637.

30. Partina I, Kalinogorskaya O, Kojima S, et al. Surveillance of antimicrobial susceptibility of Enterobacteriaceae pathogens isolated from intensive care units and surgical units in Russia. Jpn J Antibiot. 2016;69(1):41-51.

31. Pfaller MA, Shortridge D, Sader HS, et al. Ceftolozane-tazobactam activity against drug-resistant Enterobacteriaceae and Pseudomonas aeruginosa causing healthcare-associated infections in Australia and New Zealand: Report from an Antimicrobial Surveillance Program (20132015). J Glob Antimicrob Resist. 2017:186-194.

32. Logan LK, Renschler JP, Gandra S, et al. Carbapenem Resistant Enterobacteriaceae in Children, United States, 1999-2012. Emerg Infect Dis. 2015;21(11):2014-2021.

33. Sader HS, Castanheira M, Flamm RK, et al. Tigecycline activity tested against carbapenem-resistant Enterobacteriaceae from 18 European nations: results from the SENTRY surveillance program (2010-2013). Diagn Microbiol Infect Dis. 2015;83(2):183-186.

34. Almugadam BS, Elbala AS, Elkheir AS, et al. Carbapenem Resistance Enterobacteriaceae Among Wound Isolates, Kosti City, Sudan. Clin Microbiol. 2018;7:1.

35. Bouamri M, Arsalane L, Kamouni Y, et al. Antimicrobial susceptibility of urinary Klebsiella pneumoniae and the emergence of carbapenemresistant strains: A retrospective study from a university hospital in Morocco, North Africa. African Journal of Urology. 2015;21(1):36-40.

36. Zhao Z, Lan F, Liu M, et al. Evaluation of automated systems for aminoglycosides and fluoroquinolones susceptibility testing for Carbapenemresistant Enterobacteriaceae. Antimicrobial Resistance \& Infection Control. 2017;6:77.

37. Christophy R, Osman M, Mallat M, et al. Prevalence, antibiotic susceptibility and characterization of antibiotic resistant genes among carbapenem-resistant Gram-negative bacilli and yeast in intestinal flora of cancer patients in North Lebanon. J Infect and Public Health. 2017;10(6):716-720

38. Almugadam BS, Mohamed HA, Hamid HO, et al. Frequency of Carbapenem Resistance Enterobacteriaceae among Urinary Isolates in Kosti City, Sudan 2017. World J Biol Med Science. 2017;4(4):23-28

39. Nordmann P, Gniadkowski M, Giske CG, et al. Identification and screening of carbapenemase- producing Enterobacteriaceae. Clin Microbiol Infect. 2012;18(5):432-438. 
40. Kim S, Shin J, Shin S, et al. Characteristics of carbapenem-resistant Enterobacteriaceae isolates from Korea. Diagn Microbiol Infect Dis. 2013;76(4):486-490.

41. Falagas ME, Kastoris AC, Kapaskelis AM, et al. Fosfomycin for the treatment of multidrug-resistant, including extended-spectrum betalactamase producing, Enterobacteriaceae infections: A systematic review. The Lancet Infectious Diseases. 2010;10(1):43-50.

42. Petrosillo N, Giannella M, Lewis R, et al. Treatment of carbapenemresistant Klebsiella pneumoniae:the state of the art. Expert Rev Anti Infect Ther. 2013;11(2):159-177.

43. Tzouvelekis LS, Markogiannakis A, Piperaki E, et al. Treating infections caused by carbapenemase-producing Enterobacteriaceae. Clin Microbiol Infect. 2014;20(9):862-872

44. van Duin D, Kaye KS, Neuner EA, et al. Carbapenem-resistant Enterobacteriaceae: a review of treatment and outcomes. Diagn Microbiol Infect Dis. 2013;75(2):115-120.

45. Jiang X, Poirel L, Nordmann P. Lack of polymyxin resistance among carbapenemase-producing Enterobacteriaceae in a university hospital in China. Infect Dis. 2017;49(7):556-557.
46. Kelesidis T, Karageorgopoulos DE, Kelesidis I, et al. Tigecycline for the the treatment of multidrug-resistant Enterobacteriaceae: a systematic review of the evidence from microbiological and clinical studies. $J$ Antimicrob Chemother. 2008;62(5):895-904.

47. Zhan L, Wang S, Guo Y, et al. Outbreak by Hypermucoviscous Klebsiella pneumoniae ST11 Isolates with Carbapenem Resistance in a Tertiary Hospital in China. Front Cell Infect Microbiol. 2017;7:182.

48. Falagas ME, Lourida P, Poulikakosa P, et al. Antibiotic treatment of Infections Due to Carbapenem-Resistant Enterobacteriaceae: Systematic Evaluation of the Available Evidence. Antimicrob. Agents Chemother. 2014;58(2):654-663.

49. Nabarro LB, Veeraraghavan B. Combination therapy for carbapenemresistant Enterobacteriaceae: increasing evidence, unanswered questions, potential solutions. Eur J Clin Microbiol Infect Dis. 2015;34(12):23072311.

50. Fredborg M, Sondergaard TE, Wang M. Synergistic activities of meropenem double and triple combinations against carbapenemaseproducing Enterobacteriaceae. Diagn Microbiol Infect Dis. 2017; 88(4):355-360 